\title{
Impact of a Linear Array of Hydrophilic and Superhydrophobic Spheres on a Deep Water Pool
}

\author{
Guang Yang $1,2, *,+\left(\mathbb{D}\right.$, Visakh Vaikuntanathan ${ }^{2, *,+} \oplus$, Alexandros Terzis ${ }^{2}$, Xin Cheng ${ }^{1}$, \\ Bernhard Weigand ${ }^{2}$ and Rainer Helmig ${ }^{3}$ \\ 1 Institute of Refrigeration and Cryogenics, Shanghai Jiao Tong University, Shanghai 200240, China; \\ chengxin-17@sjtu.edu.cn \\ 2 Institute of Aerospace Thermodynamics (ITLR), University of Stuttgart, 70569 Stuttgart, Germany; \\ alexandros.terzis@me.com (A.T.); bernhard.weigand@itlr.uni-stuttgart.de (B.W.) \\ 3 Institute for Modelling Hydraulic and Environmental Systems, University of Stuttgart, \\ 70569 Stuttgart, Germany; rainer.helmig@iws.uni-stuttgart.de \\ * Correspondence: y_g@sjtu.edu.cn (G.Y.); visakh.vaikuntanathan@itlr.uni-stuttgart.de (V.V.) \\ + These authors contributed equally to this work.
}

Received: 23 January 2019; Accepted: 20 February 2019; Published: 22 February 2019

\begin{abstract}
The impact of solid bodies on the free surface of liquid pools is involved in many practical applications-such as bullets and air-to-sea anti-torpedo defense systems, or the water entry of athletes in water sports - aimed at improving the performance through a control of cavity dynamics. This work reports an experimental investigation of the impact of a linear array of hydrophilic $(\mathrm{H})$ and superhydrophobic (SH) spheres on a deep water pool. The array consisted of ten magnetic spheres, with different permutations of $\mathrm{H}$ and $\mathrm{SH}$ spheres. Using high speed shadowgraphy, we captured the underwater kinematics of the array for different permutations of $\mathrm{H}$ and $\mathrm{SH}$ spheres. In particular, we observed the evolution or absence of an air cavity attached to the array as a function of the position of the $\mathrm{H}$ and $\mathrm{SH}$ spheres. The position of the first $\mathrm{SH}$ sphere from the leading edge of the array $\left(\mathrm{Z}_{\mathrm{SH}}\right)$ emerged as a key parameter that alters the characteristics of cavity evolution. The appearance and pinch-off characteristics of a wake cavity behind the trailing edge were governed by the wetting properties of the leading and trailing surfaces of the array. The position of the first SH surface, as well as the wetting characteristics of the leading and trailing surfaces, are potential control parameters to alter underwater cavity evolution during solid surface impact on deep water pools.
\end{abstract}

Keywords: superhydrophobicity; water entry; fluid dynamics; wetting; experiments

\section{Introduction}

Beginning with the classical works of Worthington and Cole [1,2] more than a century ago, the impact of solid bodies on the free surface of liquid pools has been studied for a long time, motivated by practical applications-including the water entry of projectiles such as bullets and air-to-sea anti-torpedo defense systems and the water entry of athletes in water sports-aimed at improving performance through a control of cavity dynamics [3].

Experimental studies on the water entry of solid bodies have focused mainly on the effects of: (i) the size and shape of the solid body (see Figure 5 in [3]), (ii) the surface wetting and roughness characteristics of the solid body (see Figure 2 in [3]), (iii) the impact velocity and rotation of the solid body at entry (see Figure 8 in [3]), (iv) the density of the solid body material compared to that of the liquid pool (see Figure 6 in [3]), and (v) the presence of oil film on the free surface of water [4] on the dynamics of the cavity below the free surface of a liquid pool and the dynamics of the splash above the free surface of a liquid pool. 
It is known that by altering the surface wetting characteristics of solid spheres, cavity formation can be enhanced (due to more hydrophobic surfaces) or suppressed (due to more hydrophilic surfaces) $[5,6]$. The impact conditions and cavity formation are related by the $\mathrm{Ca}-\theta_{\mathrm{o}}$ phase diagram, where $\mathrm{Ca}=\mu \mathrm{V}_{\mathrm{o}} / \sigma$ is the capillary number ( $\mu$ and $\sigma$ are the dynamic viscosity and surface tension of the pool liquid), $\mathrm{V}_{\mathrm{o}}$ is the impact velocity of the solid sphere, and $\theta_{\mathrm{o}}$ is the static contact angle of the pool liquid on the solid sphere surface. At a given $\theta_{0}$, when $\mathrm{Ca}<\mathrm{Ca}_{\mathrm{cr}}$ cavity formation is suppressed, whereas when $\mathrm{Ca}>\mathrm{Ca}_{\mathrm{cr}}$ cavity formation is observed. This critical value of $\mathrm{Ca},\left(\mathrm{Ca}_{\mathrm{cr}}\right)$ is mainly a function of $\theta_{\mathrm{o}}$ and is given as follows [5]:

$$
C a_{c r}=\left\{\begin{array}{c}
\xi ; \theta_{o} \leq 90^{\circ} \\
\frac{g_{o}}{9 l \zeta}\left(\pi-\theta_{o}\right)^{3} ; \theta_{o} \geq 90^{\circ}
\end{array}\right.
$$

Here the values of the adjustable parameters that yielded best-fit with the experimental data of Duez et al. [5] were $\xi=0.1, g_{o}=7, l=15$, and $\xi=2$. Aristoff and Bush [7] further showed that there were four different cavity shapes dependent on the Bond and Weber numbers. Speirs et al. [8] indicated that the pinch-off type could be predicted by the cavity diameter and downward cavity velocity regardless of the type of impacting body.

Some recent works have also studied cavity formation characteristics in more complex conditions, such as the entry of deformable elastomeric spheres [9], entry of heated spheres [10] and entry into a water-surfactant mixture [11].

While cavity dynamics has been studied for many decades, this work focused on the effect of alternate surface wetting of solid spheres in a linear array, which has not been previously considered. The linear array consisted of hydrophilic $(\mathrm{H})$ and superhydrophobic $(\mathrm{SH})$ spheres. The rest of this paper is organized as follows: in Section 2 the experimental set-up is introduced, then in Section 3, the effect of the permutations of $\mathrm{H}$ and $\mathrm{SH}$ spheres on the appearance and pinch-off characteristics of the air cavity are analyzed in detail. The paper closes with some key conclusions in Section 4.

\section{Materials and Methods}

The experimental set-up used for studying the impact of linear arrays of spheres on a deep pool of water is schematically shown in Figure 1a. The main components were the dropper arrangement, liquid pool, and a high-speed imaging system. The dropper arrangement comprised a magnetic switch (1) attached to a long vertical tube (2) of an internal diameter slightly larger than that of the spheres, which provided directed passage for the spheres. The array of spheres were attached to the magnetic switch through a thick layer of cardboard so that when the switch was turned on, the magnet held the magnetic spheres in place. When the switch was turned off, the magnetic spheres dropped through the vertical tube made of non-magnetic material. The entire dropper arrangement was attached to a vertical traverse (3) so as to enable control of the fall height and, hence, the impact velocity. The liquid pool (4) was a glass tank with the dimensions of $300 \mathrm{~mm} \times 200 \mathrm{~mm} \times 200 \mathrm{~mm}$, filled with filtered tap water (density, $\left.\rho=1000 \mathrm{~kg} / \mathrm{m}^{3} ; \mu=1 \mathrm{mPa} \cdot \mathrm{s} ; \sigma=0.0728 \mathrm{~N} / \mathrm{m}\right)$ to a desired depth $(160 \mathrm{~mm})$. The experiments were conducted at an ambient temperature of about $25^{\circ} \mathrm{C}$.

The linear array (Figure 1b) was composed of ten magnetic spheres, each with a diameter $\left(\mathrm{D}_{\mathrm{s}}\right)$ of $5 \mathrm{~mm}$, with different combinations of hydrophilic $(\mathrm{H})$ and superhydrophobic $(\mathrm{SH})$ spheres so as to vary the position of the first superhydrophobic sphere in the array, $\mathrm{Z}_{\mathrm{SH}}$. The $\mathrm{H}$ and $\mathrm{SH}$ spheres were made of the same metallic alloy $(\mathrm{NdFeB})$ and had a density $\left(\rho_{\mathrm{s}}\right)$ of $7400 \mathrm{~kg} / \mathrm{m}^{3}$. The SH spheres were made by coating a superhydrophobic layer on $\mathrm{H}$ spheres (see [12] for details of this coating; Appendix A shows typical wetting experiments conducted on $\mathrm{H}$ and $\mathrm{SH}$ spheres). Prior to the dropping experiment, we coated over $1000 \mathrm{SH}$ spheres under the same conditions. Thus, replicate sets with fresh, previously unused spheres were used during the multiple tests. Two constant impact velocities were studied $(1 \mathrm{~m} / \mathrm{s}$ and $2 \mathrm{~m} / \mathrm{s})$ so that the effect of $Z_{\mathrm{SH}}$ on the cavity characteristics could be studied. The corresponding capillary numbers on the Duez et al. [5] Ca- $\theta_{\mathrm{o}}$ phase diagram are shown in Figure 2. 


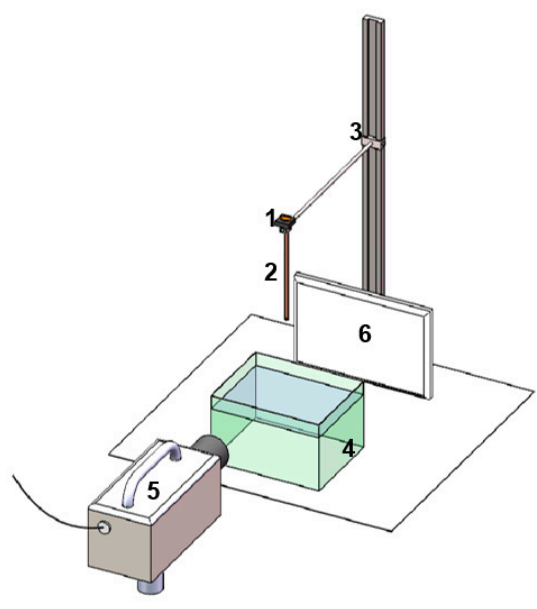

(a)

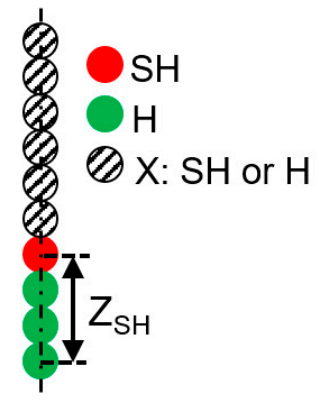

(b)

Figure 1. Schematic of (a) experimental set-up highlighting its major components: (1) magnetic switch; (2) long vertical tube; (3) vertical traverse; (4) liquid pool; (5) CCD camera; and (6) diffused backlighting. (b) The linear array of spheres defining the parameter $Z_{\mathrm{SH}}$ which characterizes the position of the first SH sphere.

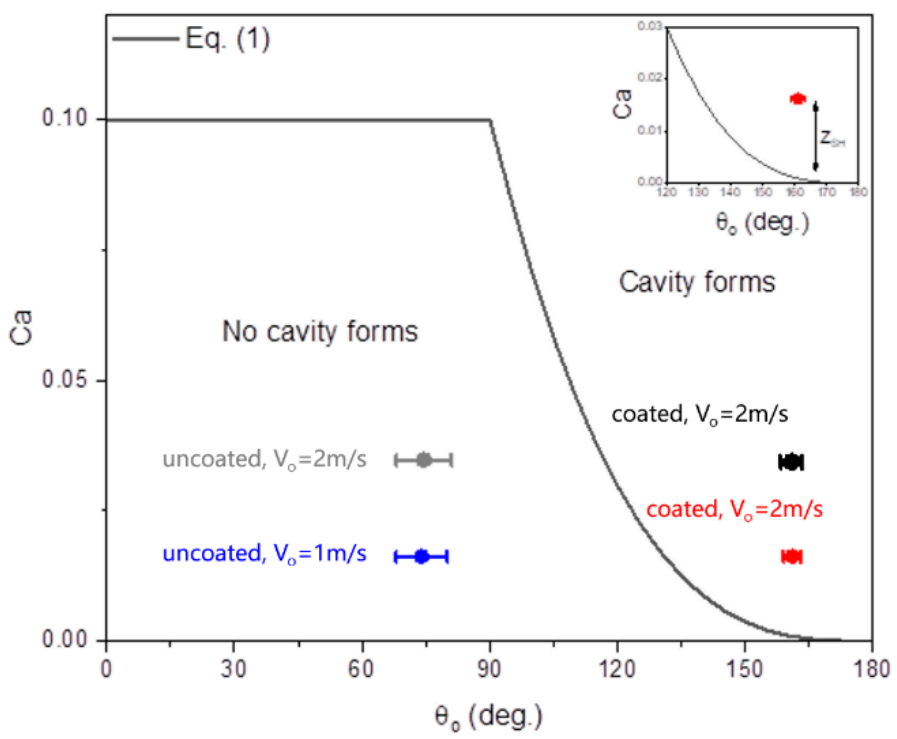

Figure 2. Ca versus $\theta_{\mathrm{o}}$ map showing the critical Ca for cavity formation as given for a single sphere by Equation (1) [5]; the values of $\theta_{\mathrm{o}}$ for $\mathrm{H}$ (uncoated) and $\mathrm{SH}$ (coated) spheres are $\approx 74^{\circ}$ (see Figure A1) and $\approx 161^{\circ}$ [12], respectively. (Inset) Magnification of the SH case illustrating a control of Ca with $\mathrm{Z}_{\mathrm{SH}}$.

The high-speed imaging system consisted of a Photron FASTCAM SAX-2 camera (5) along with diffused backlighting (6). The operating parameters of the high-speed camera were kept constant for all the experiments: frame rate $=12,500 \mathrm{fps}$, spatial resolution $=0.125 \mathrm{~mm} /$ pixel, and exposure $=$ 0.078 milliseconds.

\section{Results and Discussion}

\subsection{Cavity Evolution}

Figure 3 shows the evolution of the cavities beneath the water surface for four cases of linear arrays of $\mathrm{H}$ and $\mathrm{SH}$ spheres differing in the position of the first $\mathrm{SH}$ sphere, with the rest of each array made of $\mathrm{H}$ spheres, from top to bottom: $\mathrm{Z}_{\mathrm{SH}}=0 \mathrm{~mm}, 5 \mathrm{~mm}, 15 \mathrm{~mm}$, and $45 \mathrm{~mm}$. Note that the images 
were chosen at appropriate time instants to highlight cavity evolution. For $Z_{\mathrm{SH}}=0 \mathrm{~mm}$, the cavity formed at the leading edge of the linear array. It expanded radially outward, reaching a maximum width ( $\left.W_{\max }\right)$ in the process (second and third frames). It then receded radially towards the array axis, collapsing between the sixth and seventh spheres in the array (fourth and fifth frames). This is referred to as the 'first pinch-off' event. It was characterized by (i) the first pinch-off time $\left(t_{p 1}\right)$ defined as the corresponding time elapsed after the array first contacts the free surface of the water $(t=0)$; (ii) the relative first pinch-off time $\left(t_{p 1}-t_{f 1}\right)$, where $t_{f 1}$ is the instant at which the cavity starts forming; and (iii) the first pinch-off depth $\left(\mathrm{H}_{\mathrm{p} 1}\right)$, defined as the depth of the first pinch-off location from the free surface of the water. Afterwards, as the array kept falling through the pool, the upper part of the pinched-off cavity underwent a second pinch-off in the wake of the last sphere in the array (sixth and seventh frames). This second pinch-off event was characterized by (i) the second pinch-off time $\left(t_{\mathrm{p} 2}\right)$, and (ii) the second pinch-off depth $\left(\mathrm{H}_{\mathrm{p} 2}\right)$, both defined similarly to those of the first pinch-off. This was sharply in contrast with the cavity evolution for the single SH sphere impact (see Figure A2b), which exhibited only one cavity pinch-off event. For more details regarding the cavity formation and evolution characteristics, please refer to our videos in the Gallery of Fluid Motion [13].

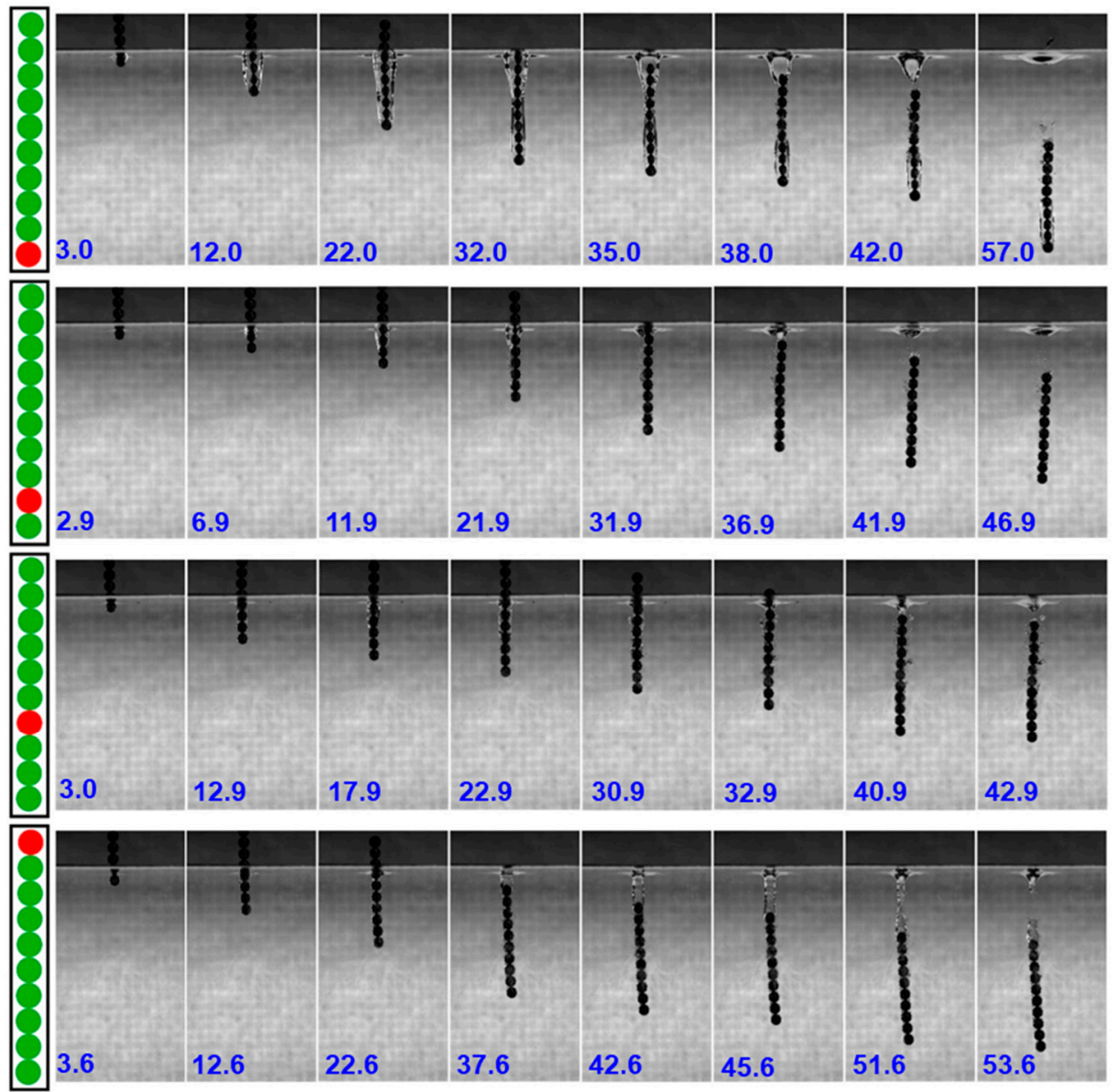

Figure 3. Images showing the time evolution of the impact process showing the effect of $Z_{\mathrm{SH}}$ on the cavity evolution characteristics $\left(\mathrm{V}_{0}=1 \mathrm{~m} / \mathrm{s}\right)$. From top to bottom, $\mathrm{Z}_{\mathrm{SH}}=0 \mathrm{~mm}, 5 \mathrm{~mm}, 15 \mathrm{~mm}$, and 45 $\mathrm{mm}$. The time instant (in milliseconds) of each frame is shown at its bottom left corner.

As the position of the first $\mathrm{SH}$ sphere shifted away from the leading edge $\left(\mathrm{Z}_{\mathrm{SH}}=0 \mathrm{~mm}\right)$ to $\mathrm{Z}_{\mathrm{SH}}=$ $5 \mathrm{~mm}$ (second row in Figure 3), even though the qualitative characteristics of cavity formation and evolution remained similar to that at $Z_{\mathrm{SH}}=0 \mathrm{~mm}$, there was a drastic difference in the quantitative characteristics. As the second sphere in the array entered the pool surface, the cavity began to form (second frame), however it expanded to less radial and axial extents than were observed at $\mathrm{Z}_{\mathrm{SH}}=0$ 
$\mathrm{mm}$ (third frame) and collapsed faster (fourth frame). As the array fell through the pool, a smaller air cavity, which was a residue of the upper part of the pinched-off first cavity, was seen at the wake of the last sphere (fifth and sixth frames) and pinched off afterwards (seventh frame). The size of this wake cavity was much smaller than that observed at $Z_{\mathrm{SH}}=0 \mathrm{~mm}$. Further, as $Z_{\mathrm{SH}}$ was increased to $15 \mathrm{~mm}$ (third row in Figure 3), the cavity started to form when the fourth sphere in the array entered the water pool. These cavity dimensions were found to be smaller (third and fourth frames) and it pinched off much faster (fifth frame) than in case of $Z_{\mathrm{SH}}=0 \mathrm{~mm}$. The residual air cavity from this pinch-off at the wake of the last sphere (sixth and seventh frames) was also smaller than at $Z_{\mathrm{SH}}=0 \mathrm{~mm}$. The last row in Figure 3 presents the case when the first $\mathrm{SH}$ sphere is the last sphere in the array $\left(Z_{\mathrm{SH}}=45 \mathrm{~mm}\right)$. There was no cavity formed until the last sphere entered the water pool (fourth frame). The cavity which then formed at the wake of the last sphere was longer than the wake cavities observed in the previous cases and narrower than the one formed in the condition $\mathrm{Z}_{\mathrm{SH}}=0 \mathrm{~mm}$ (first row in Figure 3). This discussion clearly demonstrates that by changing the position of the first $\mathrm{SH}$ sphere in the array, the characteristics of cavity evolution can be controlled.

\subsection{Maximum Cavity Width}

The quantitative characteristics of the cavities observed above were extracted for different combinations of the position of the first $\mathrm{SH}$ sphere. Figure 4 shows quantitative trends of the maximum cavity width as a function of the position of the first $\mathrm{SH}$ sphere in the array. The length scales were normalized with the diameter of the sphere, $D_{s}$. Figure 4 a shows that the maximum cavity width decreased as $Z_{\mathrm{SH}}$ increased. This can be explained as follows: as $\mathrm{Z}_{\mathrm{SH}}$ increased, the flow velocity $(\mathrm{V})$ encountered by the first SH sphere decreased due to (i) the position of the sphere at the wake of the flow around the upstream sphere, and (ii) the absence of a strong stagnation point flow compared to that at $Z_{S H}=0$. This decrease in the flow velocity led to a decrease in the effective capillary number $\left(\mathrm{Ca}_{\mathrm{eff}}=\right.$ $\mu \mathrm{V} / \sigma$ ). In Figure 2, if we used $\mathrm{Ca}_{\text {eff }}$ instead of $\mathrm{Ca}$, the decrease in $\mathrm{Ca}_{\text {eff }}$ at a given $\theta_{\mathrm{o}}$ (of an SH sphere) due to the increase in $\mathrm{Z}_{\mathrm{SH}}$, led to a less dominant cavity formation (as illustrated in the inset of Figure 1). This is quantitatively captured in Figure $4 \mathrm{~b}$ by the decrease in maximum cavity width with increasing $\mathrm{Z}_{\mathrm{SH}}$. It should be noted that the results of two limit cases, namely $10 \mathrm{SH}$ spheres and $10 \mathrm{H}$ spheres, are also included this figure, and were within the error bars of $Z_{S H} / D_{S}=0$ and $Z_{S H} / D_{s}=9$, respectively.

It is interesting to note that the maximum cavity width for the impact of a single SH sphere was the same as that for the impact of a linear array of spheres when the leading sphere was superhydrophobic $\left(Z_{\mathrm{SH}}=0\right)$. An increase of maximum cavity width due to the increase of impact velocity was also clearly observed. At higher values of $Z_{S H}$, this difference due to impact velocity became less significant (note that when $Z_{\mathrm{SH}}=9 \mathrm{D}_{\mathrm{s}}$, both the velocity cases merged).

\subsection{First Pinch-Off}

The time scales were normalized with the characteristic time scale of a sphere of diameter $D_{s}$ falling under gravitational acceleration $(\mathrm{g})$ given by $\left(2 \mathrm{~g} / \mathrm{D}_{\mathrm{s}}\right)^{0.50}[3]$. The relative pinch-off time of the first cavity $\left(t_{\mathrm{p} 1}-t_{\mathrm{f} 1}\right.$ ) decreased as $\mathrm{Z}_{\mathrm{SH}}$ increased (Figure $5 \mathrm{a}$ ), since the maximum cavity width decreased, whereas the cavity collapse rate, governed mainly by the physical properties of the pool liquid at a given impact velocity, remained the same. Note that the absolute pinch-off time $\left(t_{p 1}\right)$ showed an initial decrease between $Z_{S H} / D_{s}=0$ and $Z_{S H} / D_{s}=1$, and then increased afterwards, whereas the cavity formation instant $\left(t_{f 1}\right)$ increased with $Z_{S H}$ (see inset of Figure $5 a$ ). Moreover, the pinch-off time when the first sphere was an SH was close to the one for the impact of a single SH sphere from present study as well as to that reported in literature $(\approx 1.73 \pm 0.07)$ [3]. The impact velocity was also found to have little effect on pinch-off time. This is because both the maximum cavity width and the cavity collapse rate increased with the impact velocity. 
(a)
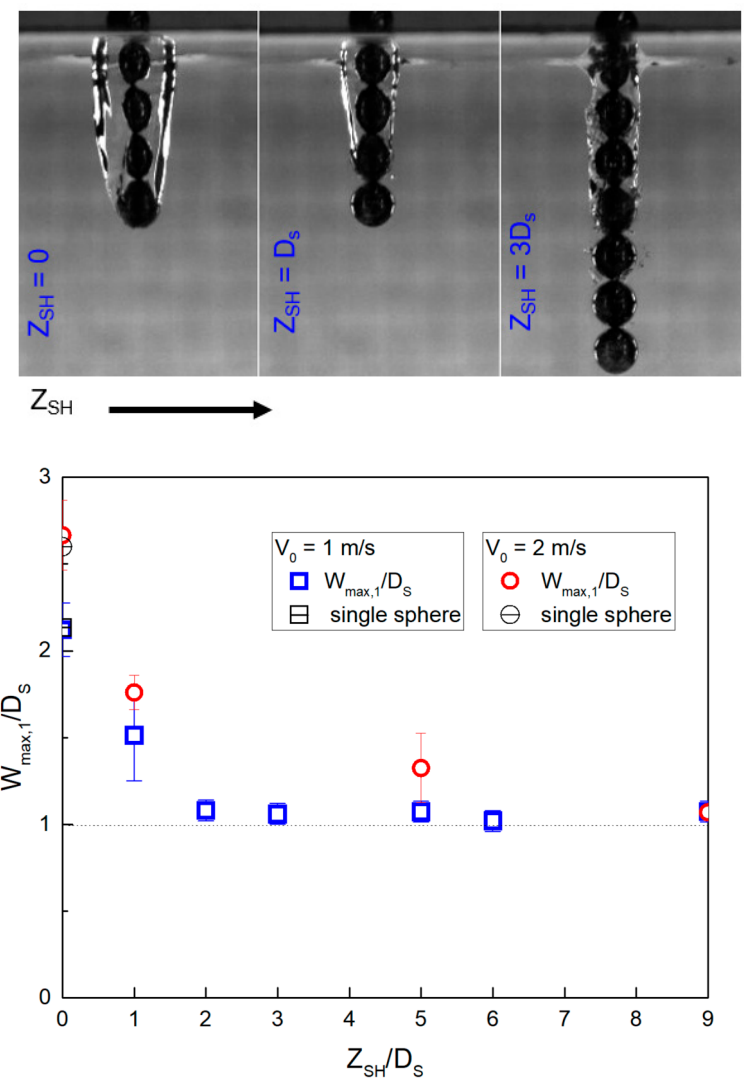

Figure 4. Maximum cavity width: (a) Images showing the cavity width at different $Z_{S H}$ values $\left(V_{\mathrm{o}}=1 \mathrm{~m} / \mathrm{s}\right)$, (b) A plot showing normalized maximum cavity width, $\mathrm{W}_{\max } / \mathrm{D}_{\mathrm{s}}$ versus $\mathrm{Z}_{\mathrm{SH}} / \mathrm{D}_{\mathrm{s}}$.

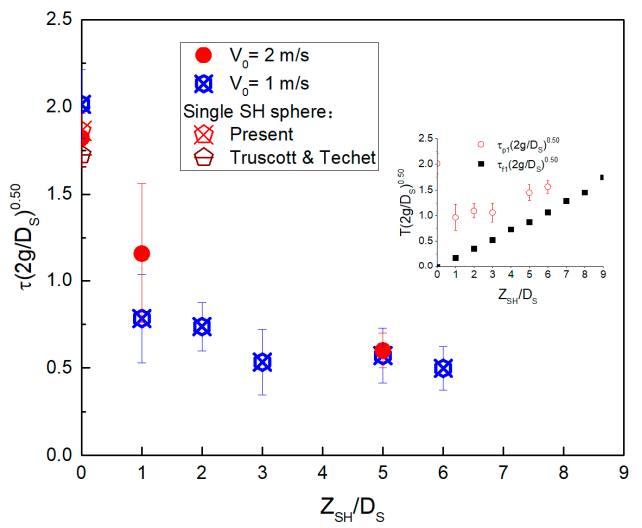

(a)

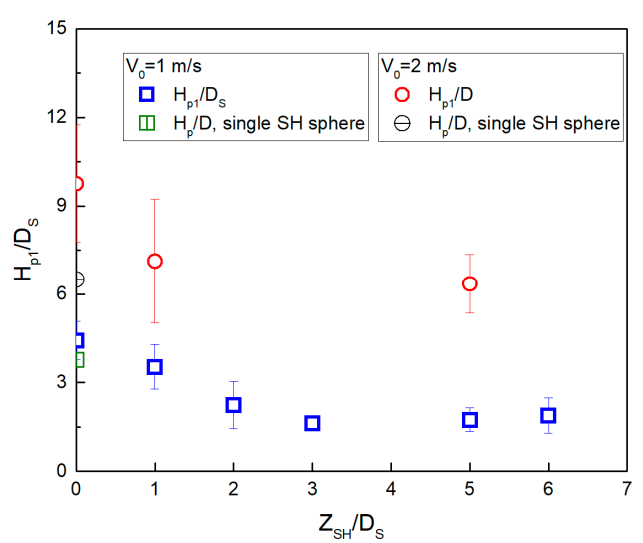

(b)

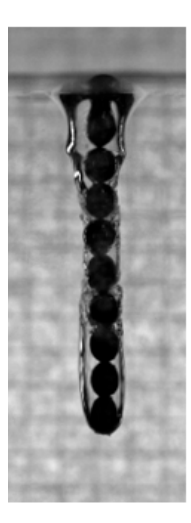

Figure 5. First pinch-off: (a) Variation of normalized relative pinch-off time, $\left(t_{p 1}-t_{f 1}\right)\left(2 g / D_{s}\right)^{0.50}$ with $\mathrm{Z}_{\mathrm{SH}} / \mathrm{D}_{\mathrm{s}}$. (Inset) The variation of normalized pinch-off time, $\mathrm{t}_{\mathrm{p} 1}\left(2 \mathrm{~g} / \mathrm{D}_{\mathrm{s}}\right)^{0.50}$ and cavity formation time, $t_{f 1}\left(2 g / D_{s}\right)^{0.50}$ with $Z_{S H} / D_{s}$. (b) Variation of normalized pinch-off depth, $H_{p 1} / D_{s}$ with $Z_{S H} / D_{s}$. (The image on the right shows a typical cavity profile when the first pinch-off happens).

The depth from the free surface of the pool at which the first pinch-off occurred $\left(\mathrm{H}_{\mathrm{p} 1}\right)$, also decreased as $Z_{S H}$ increased (Figure $5 b$ ). This was mainly due to the decrease in relative pinch-off time which, at a given impact velocity, led to a decrease in the distance or depth travelled by the cavity-forming sphere during this time. The pinch-off depth for the impact of a single SH sphere was very close to that observed when $Z_{\mathrm{SH}}=0$. The trend of the first pinch-off depth with $Z_{\mathrm{SH}}$ for the higher impact velocity case was similar. However, the values of $\mathrm{H}_{\mathrm{p} 1}$ at $2 \mathrm{~m} / \mathrm{s}$ were larger than that at $1 \mathrm{~m} / \mathrm{s}$; this was because the distance/depth travelled between cavity formation and first pinch-off 
times increased with impact velocity. These results demonstrated that $Z_{S H}$ is a new parameter, in addition to the properties of the pool liquid, impact velocity, wetting characteristics, and size/shape of the solid surface, to control the evolution characteristics of the first cavity.

\subsection{Second Pinch-Off}

The second pinch-off characteristics were governed more by the wetting properties of the first and last spheres (as discussed with Figure 3). Hence, in Figure 6a, the impact cases were grouped into four as follows: the first and last spheres were hydrophilic (Case I/H-H), the first sphere was superhydrophobic and the last sphere was hydrophilic (Case II/SH-H), the first and last spheres were superhydrophobic (Case III/SH-SH), finally the first sphere was hydrophilic and the last sphere was superhydrophobic (Case IV/H-SH). Cases I, II and IV correspond respectively to conditions similar to those in second and third rows, first row, and fourth row of Figure 3.

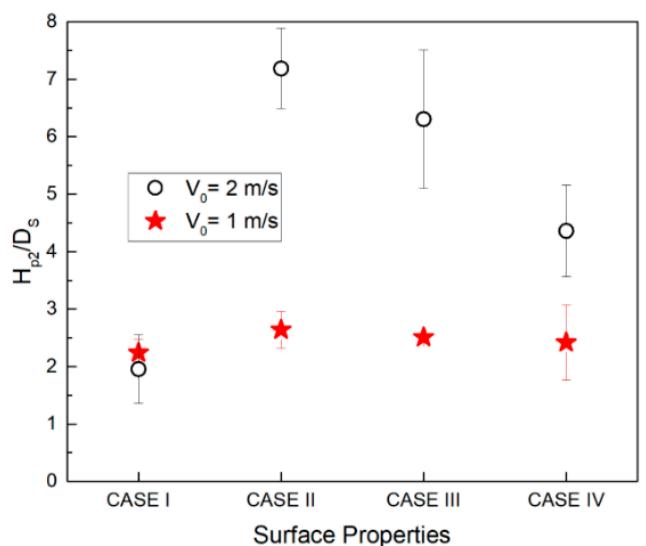

(a)

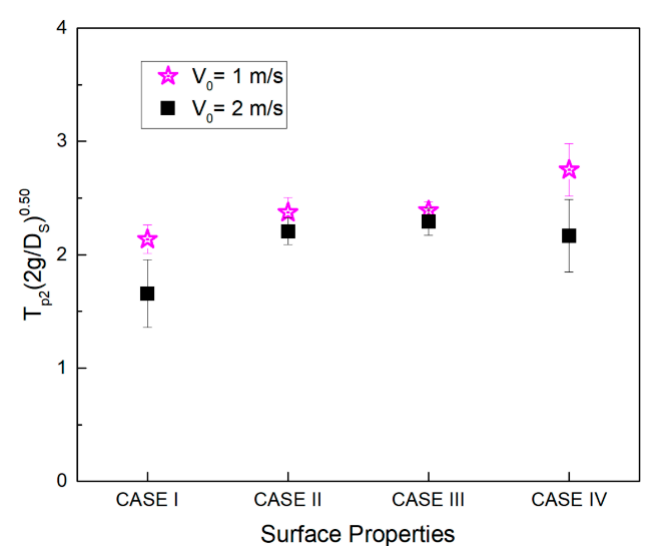

(b)

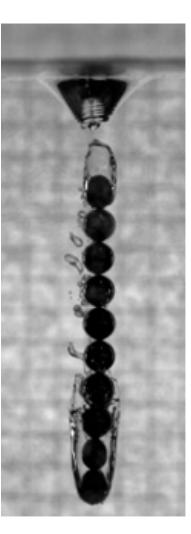

Figure 6. Second pinch-off: (a) Normalized pinch-off time, tp2(2g/Ds)0.50 and (b) normalized pinch-off depth, Hp2/Ds of the wake cavity for four categories of linear arrays based on the wetting of first and last spheres in the array. Case I: H/H. Case II: SH/H. Case III: SH/SH. Case IV: H/SH. (The image on the right shows a typical cavity profile when the second pinch-off happens).

When the first and last spheres were hydrophilic (Case I/H-H), the wake cavity was the least pronounced and hence the pinch-off time and depth were the smallest (see Figure 6). This was because of the less dominant effect of first cavity pinch-off due to the presence of the first hydrophilic sphere and inability to form a dominant wake cavity by the last hydrophilic sphere. When the first sphere in Case I/H-H was replaced with a superhydrophobic sphere (Case II/SH-H), the wake cavity became more dominant (longer pinch-off time and greater depth) due to the dominant pinch-off of the first cavity which led to a prominent upper part of the pinched-off cavity interacting with the last sphere at its wake. When both the first and last spheres in Case I/H-H were replaced with superhydrophobic spheres (Case III/SH-SH), the wake cavity pinch-off was similar to Case II/SH-H. This is probably because the development of wake cavity was affected more dominantly by the pinch-off of the first cavity. When the last sphere in Case I/H-H was replaced with a superhydrophobic sphere (Case $\mathrm{IV} / \mathrm{H}-\mathrm{SH}$ ), the wake cavity became more dominant than Case I/H-H (larger pinch-off time and depth). Similar to the first pinch-off, the higher impact velocity led to a higher second pinch-off depth, but had little effect on the pinch-off time.

The above discussion indicates that when the first sphere was hydrophilic, the wake cavity was more sensitive to the wetting characteristics of the last sphere; whereas when the first sphere was superhydrophobic, the wake cavity was not affected much by the wetting characteristics of the last sphere. Hence tuning the wetting characteristics of the first and last spheres in the array provided a new pathway to control the wake cavity. 


\section{Conclusions}

To summarize, we presented a preliminary study on the impact of a linear array of hydrophilic and superhydrophobic spheres on a pool of water. Comparison of the impact of single superhydrophobic and linear array of hydrophilic and superhydrophobic spheres showed the presence of two pinch-off events corresponding to the first and wake cavities in the linear array case. Through systematic experiments and analysis, we demonstrated that by controlling the position of the superhydrophobic surface in the array, the evolution characteristics of the first cavity could be controlled. Furthermore, by varying the wetting properties of the leading and trailing edge surfaces, the pinch-off characteristics of the wake cavity were able to be fine-tuned.

This study showed that-in addition to the conventional parameters such as impact velocity, wetting characteristics, and size/shape of the solid surface-the position of the non-wetting surface and the wetting characteristics of the leading and trailing surfaces offer a new pathway to control the evolution characteristics of the cavities formed during the water entry of solid surfaces. Future work in this direction will involve exploring the effects of the impact velocity and the size of the array (length, diameter, and aspect ratio) on the efficacy of $Z_{\mathrm{SH}}$ as a control parameter, especially at higher impact velocities and larger sizes of array, in underwater cavity dynamics as well as the splash characteristics above the free surface of the pool.

Author Contributions: V.V., G.Y., and A.T. conceived the idea. V.V., G.Y., and X.C. performed the experiments, analysed the data, and wrote the draft manuscript. A.T., B.W. and R.H. provided feedback and suggestions on the experiments and draft manuscript. All the authors read and approved the manuscript.

Funding: The authors acknowledge the financial support from the German Research Foundation (DFG) through the International Research Training Group (GRK 2160/1) Droplet Interaction Technologies (DROPIT) and SFB 1313, Research Project A02. A. T. acknowledges the Alexander von Humboldt (AvH) foundation for the funding support.

Conflicts of Interest: The authors declare no conflict of interest.

\section{Appendix A}
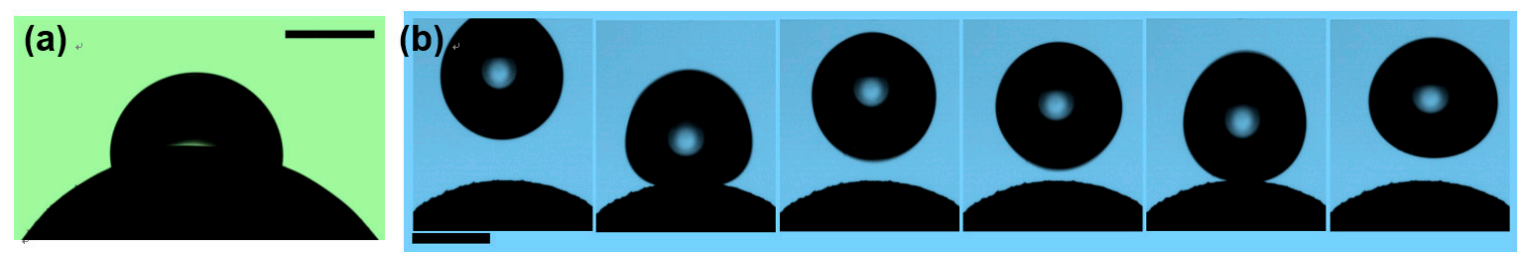

Figure A1. Interaction of water droplets with (a) uncoated, and (b) coated spheres. These experiments were conducted using a standard contact angle goniometer (DataPhysics OCA15EC). (a) On the uncoated sphere, when gently placed, the water droplet formed a sessile droplet with contact angle, $\theta_{\mathrm{o}}=74.1^{\circ} \pm 6.0^{\circ}$. (b) On the coated sphere, it was not possible to gently deposit a water droplet since the needle tip was always more hydrophilic than the coated surface of the sphere. This resulted in the droplet gently bouncing off the surface even at the smallest possible height of the needle from the surface of the sphere at which droplet detached from the needle before contacting the sphere surface. Time increases from left to right in (b), which shows two consecutive bounce-off events. The scale bars shown in the images correspond to a length of $1 \mathrm{~mm}$. 


\section{Appendix B}
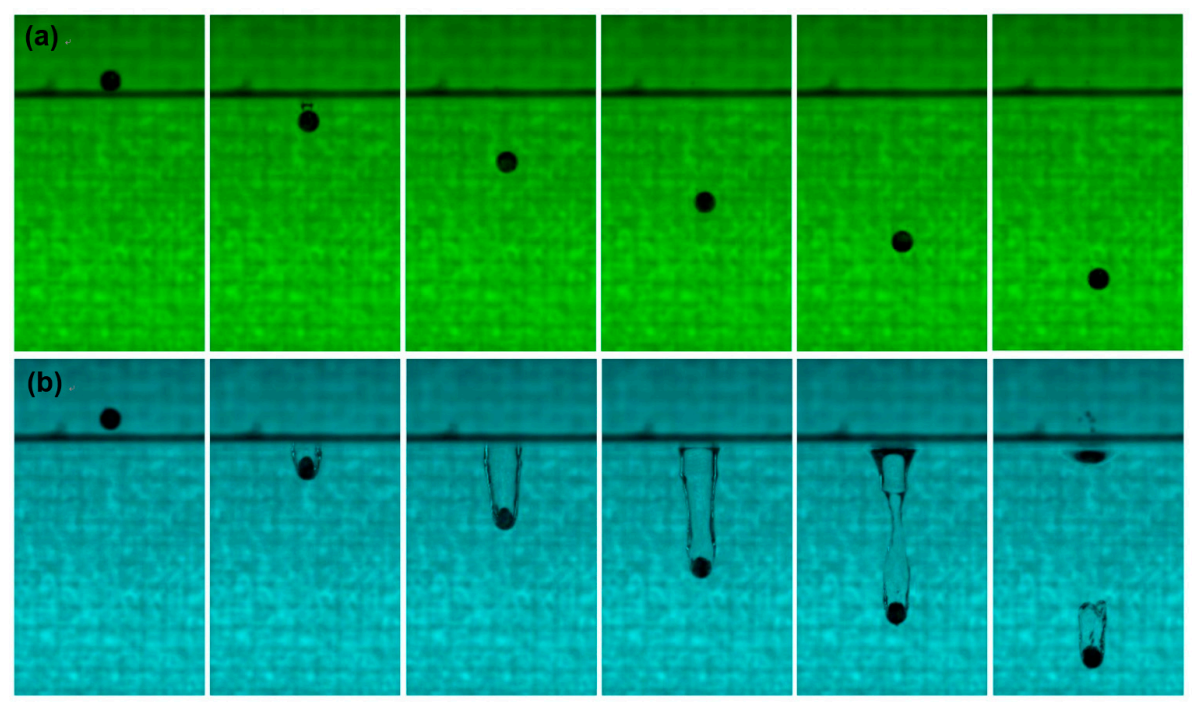

Figure A2. Interactions of (a) uncoated and (b) coated spheres $5.0 \mathrm{~mm}$ in diameter, $\left(\mathrm{D}_{\mathrm{s}}\right)=$ with a pool of water at an impact velocity, $V_{O}=1.00 \mathrm{~m} / \mathrm{s}( \pm 0.09 \mathrm{~m} / \mathrm{s})$. (a) The uncoated sphere impacts on the free surface of the pool (just below the thick dark line) resulting in a small air bubble in its wake (second frame) that is not seen as the sphere falls through the pool. This shows the hydrophilic nature of the uncoated sphere surface. (b) The coated sphere impacts on the free surface of the pool, resulting in the formation of an air cavity in its wake (second frame), which elongates as the sphere falls through the pool (third and fourth frames), radially shrinking in the process (fourth and fifth frames), and finally pinching off (last frame) with one part still attached to the wake of the sphere, whereas the other part resulted in the Worthington jet on the free surface (not studied here). The time difference between two consecutive frames in the image sequence was 8.0 milli-seconds.

\section{References}

1. Worthington, A.M.; Cole, R.S. Impact with a liquid surface, studied by the aid of instantaneous photography. Phil. Trans. A 1897, 189, 137-148. [CrossRef]

2. Worthington, A.M.; Cole, R.S. Impact with a liquid surface, studied by the aid of instantaneous photography-Paper II. Phil. Trans. A 1900, 194, 175-200. [CrossRef]

3. Truscott, T.T.; Epps, B.P.; Belden, J. Water entry of projectiles. Annu. Rev. Fluid Mech. 2014, 46, $355-378$. [CrossRef]

4. Tan, B.C.W.; Thomas, P.J. Influence of an upper layer liquid on the phenomena and cavity formation associated with the entry of solid spheres into a stratified two-layer system of immiscible liquids. Phys. Fluids 2018, 30, 064104. [CrossRef]

5. Duez, C.; Ybert, C.; Clanet, C.; Bocquet, L. Making a splash with water repellency. Nat. Phys. 2007, 3, $180-183$. [CrossRef]

6. Truscott, T.T.; Techet, A.H. A spin in cavity formation during water entry of hydrophobic and hydrophilic spheres. Phys. Fluids 2009, 21, 121703. [CrossRef]

7. Aristoff, J.M.; Bush, J.W. Water entry of small hydrophobic spheres. J. Fluid Mech. 2009, 619, 45-78. [CrossRef]

8. Speirs, N.B.; Mansoor, M.M.; Belden, J.; Truscott, T.T. Water entry of spheres with various contact angles. J. Fluid Mech. 2019, 862, 3. [CrossRef]

9. Hurd, R.C.; Belden, J.; Jandron, M.A.; Fanning, D.T.; Bower, A.F.; Truscott, T.T. Water entry of deformable spheres. J. Fluid Mech. 2017, 824, 912-930. [CrossRef]

10. Li, J.C.; Wei, Y.J.; Wang, C.; Xia, W.X. Cavity formation during water entry of heated spheres. Chin. Phys. B 2018, 27, 094703. [CrossRef] 
11. Speirs, N.B.; Mansoor, M.M.; Hurd, R.C.; Sharker, S.I.; Robinson, W.G.; Williams, B.J.; Truscott, T.T. Entry of a sphere into a water-surfactant mixture and the effect of a bubble layer. Phys. Rev. Fluids 2018, 3, 104004. [CrossRef]

12. Gupta, R.; Vaikuntanathan, V.; Sivakumar, D. Superhydrophobic qualities of an aluminum surface coated with hydrophobic solution NeverWet. Colloid Surf. A 2016, 500, 45-53. [CrossRef]

13. Yang, G.; Vaikuntanathan, V.; Terzis, A.; Weigand, B.; Helmig, R. Impact of a linear array of hydrophilic and superhydrophobic spheres on water pool. In Gallery of Fluid Motion, 71st Annual Meeting of the APS Division of Fluid Dynamics, Atlanta, GA, USA, 18-20 November 2018; The Division of Fluid Dynamics of the American Physical Society: College Park, MD, USA, 2018. [CrossRef]

2019 by the authors. Licensee MDPI, Basel, Switzerland. This article is an open access article distributed under the terms and conditions of the Creative Commons Attribution (CC BY) license (http://creativecommons.org/licenses/by/4.0/). 